Proceedings of the

International Geometry Center

Vol. 10, no. 2 (2017) pp. 39-46

\title{
Властивості спряжених функцій у гіперкомплексному просторі
}

\author{
М. В. Стефанчук
}

\begin{abstract}
We introduce new notions of linearly convex and conjugated functions in multidimensional hypercomplex space being a $n$-product of quaternions. There are investigated properties of these objects.
\end{abstract}

Анотація. В даній роботі вводиться поняття лінійно опуклих та спряжених функцій у $n$-вимірному гіперкомплексному просторі $\mathbb{H}^{n}$, досліджуються їхні властивості.

\section{1. ЛІнІйно опУкЛІ ФУнкцї̈}

В даній роботі ми узагальнюємо деякі результати щодо багатозначних функцій у комплексному просторі $\mathbb{C}^{n}$ (див. [1]) на $n$-вимірний гіперкомплексний простір $\mathbb{H}^{n}, n=1,2, \ldots$, що є прямим добутком $n$ копій тіла кватерніонів $\mathbb{H}\left(\mathbb{H}^{1}:=\mathbb{H}\right)[2],[3]$. Для довільної пари

$$
x=\left(x_{1}, \ldots, x_{n}\right), \quad y=\left(y_{1}, \ldots, y_{n}\right) \in \mathbb{H}^{n}
$$

визначимо скалярний добуток $\langle x, y\rangle$ за формулою:

$$
\langle x, y\rangle:=\sum_{m=1}^{n} x_{m} y_{m} .
$$

Означення 1.1. Функція $f: \mathbb{H}^{n} \rightarrow \mathbb{H}$ називається багатозначною, якщо образом точки $x \in \mathbb{H}^{n} \in$ множина $f(x) \in \mathbb{H}$.

Область визначення такої функції будемо позначати через

$$
E_{f}:=\left\{x \in \mathbb{H}^{n} \mid \text { існує таке } y \in \mathbb{H}, \text { що } y=f(x)\right\} .
$$

Означення 1.2. Функція $l: \mathbb{H}^{n} \rightarrow \mathbb{H}$ називається афінною, якщо її графіком є гіперплощина.

Ключові слова: багатозначна функція, афінна функція, лінійно опукла функція, спряжена функція

УДК $514.17,517.55$ 
Означення 1.3. Багатозначна функція $f: \mathbb{H}^{n} \rightarrow \mathbb{H}$ називається лінійно опуклою, якщо для довільної пари точок $\left(x_{0}, y_{0}\right) \in\left(\mathbb{H}^{n} \times \mathbb{H}\right) \backslash \Gamma(f)$ існує афінна функція $l: \mathbb{H}^{n} \rightarrow \mathbb{H}$, така, що $y_{0}=l\left(x_{0}\right)$ і $\Gamma(l) \cap \Gamma(f)=\varnothing$ для всіх $x \in \mathbb{H}^{n}$, де через $\Gamma(l)$ та $\Gamma(f)$ позначено графіки функцій $l$ та $f$ відповідно.

Означення 1.4. Лінійно угнутою функцією називається така багатозначна функція $f: \mathbb{H}^{n} \rightarrow \mathbb{H}$, для якої функція $\varphi=\mathbb{H} \backslash f$ е лінійно опуклою.

Це означає, що $\mathbb{H}^{n+1} \backslash \Gamma(f)$ є графіком лінійно опуклої функції, тобто що через кожну точку $\left(x_{0}, y_{0}\right) \in \Gamma(f)$ проходить графік афінної функції, який повністю міститься у $\Gamma(f)$.

Означення 1.5. Багатозначною афінною функиією називається функція, лінійно опукла і лінійно угнута одночасно, і для якої знайдеться принаймні одна точка $x \in \mathbb{H}^{n}$, в якій кожна з множин $f(x) \cap \mathbb{H}$ та $\mathbb{H} \backslash f(x)$ є непорожньою.

Означення лінійно опуклої функції можна поширити на багатозначні функції, які приймають значення в розширеній гіперкомплексній площині $\mathbb{H}^{o}=\mathbb{H} \cup\{\infty\}$, компактифікованій однією точкою.

Наведемо деякі приклади лінійно опуклих функцій.

Означення 1.6. Функція

$$
W_{E}(y)=\mathbb{H}^{o} \backslash \cup_{x \in E}\langle x, y\rangle
$$

називається опорною функцією множини $E \subset \mathbb{H}^{n}$.

Означення 1.7. Якщо $E \subset \mathbb{H}^{n}-$ лінійно опукла множина, то функція

$$
\delta_{E}(x)= \begin{cases}0, & \text { якщо } x \in E, \\ \infty, & \text { якщо } x \notin E,\end{cases}
$$

називається її індикаторною функиією.

Легко переконатися, що опорна та індикаторна функції лінійно опуклі.

Теорема 1.8. Якщо $f_{\alpha}, \alpha \in A$, е сім'єю лінійно опуклих функиій, де $A$-довільна множина індексів, то функція $f=\bigcap_{\alpha \in A} f_{\alpha}$ е лінійно опуклою.

Доведення. Маємо $\Gamma(f)=\bigcap_{\alpha \in A} \Gamma\left(f_{\alpha}\right)$. Візьмемо довільну точку

$$
\left(x_{0}, y_{0}\right) \in\left(\mathbb{H}^{n} \times \mathbb{H}\right) \backslash \Gamma(f)=\left(\mathbb{H}^{n} \times \mathbb{H}\right) \backslash \underset{\alpha \in A}{\bigcap} \Gamma\left(f_{\alpha}\right) .
$$


Тоді

$$
\left(x_{0}, y_{0}\right) \in\left(\mathbb{H}^{n} \times \mathbb{H}\right) \backslash \Gamma\left(f_{\alpha}\right)
$$

для деякого $\alpha$, а тому існує афінна функція $l: \mathbb{H}^{n} \rightarrow \mathbb{H}$, графік якої не перетинає $\Gamma\left(f_{\alpha}\right)$. Тому він тим більше не перетинає $\Gamma(f)$. Отже, функція $f \in$ лінійно опуклою.

\section{2. СПРЯЖЕНІ ФУНКЦіЇ}

Означення 2.1. Функцією, спряженою з $f$, називається функція, що задається рівністю

$$
f^{*}(y)=\mathbb{H}^{o} \backslash \underset{x \in \mathbb{H}^{n}}{\cup}(\langle x, y\rangle-f(x)) .
$$

3 означення спряженої функції випливає гіперкомплексний аналог нерівності Юнга-Фенхеля [1]:

$$
\langle x, y\rangle \notin f(x)+f^{*}(y) .
$$

Співвідношення (2.2) можна переписати у вигляді

$$
\langle x, y\rangle \in \mathbb{H} \backslash\left(f(x)+f^{*}(y)\right)
$$

або

$$
f(x) \cap\left(\langle x, y\rangle-f^{*}(y)\right)=\varnothing
$$

при всіх $x, y \in \mathbb{H}^{n}$.

Знайдемо функцію, спряжену до функції $f^{*}$ :

$$
f^{* *}(x)=\left(f^{*}\right)^{*}(x)=\mathbb{H}^{o} \backslash \bigcup_{y \in \mathbb{H}^{n}}\left(\langle x, y\rangle-f^{*}(y)\right) .
$$

Приклад 2.2. Спряженою з багатозначною афінною функцією

$$
f(x)=\left\langle x, y_{0}\right\rangle+f(\Theta),
$$

де $f(\Theta) \subset \mathbb{H}$ - множина, яка є образом точки $\Theta=(0,0, \ldots, 0) \in \mathbb{H}^{n}, \epsilon$ функція

$$
\begin{aligned}
f^{*}(y) & =\mathbb{H}^{o} \backslash \bigcup_{x \in \mathbb{H}^{n}}\left(\langle x, y\rangle-\left\langle x, y_{0}\right\rangle-f(\Theta)\right)= \\
& =\mathbb{H}^{o} \backslash \bigcup_{x \in \mathbb{H}^{n}}\left(\left\langle x, y-y_{0}\right\rangle-f(\Theta)\right)= \\
& = \begin{cases}\mathbb{H}^{o} \backslash(-f(\Theta)), & \text { якщо } y=y_{0}, \\
\infty, & \text { якщо } y \neq y_{0} .\end{cases}
\end{aligned}
$$

Приклад 2.3. Нехай $E \subset \mathbb{H}^{n}, \mathbb{H}^{n} \backslash E \neq \varnothing$ i $f(x)=\delta_{E}(x)$. Тоді

$$
f^{*}(y)=\mathbb{H}^{o} \backslash \bigcup_{x \in \mathbb{H}^{n}}\left(\langle x, y\rangle-\delta_{E}(x)\right)=\mathbb{H}^{o} \backslash \bigcup_{x \in \mathbb{H}^{n} \subset E}\langle x, y\rangle,
$$

тобто спряженою з індикаторною функцією власної підмножини $E$ буде опорна функція цієї множини. 
Теорема 2.4. Для кожної багатозначної функиї $f: \mathbb{H}^{n} \rightarrow \mathbb{H}$ справедливе включення $f \subset f^{* *}$.

Доведення. Візьмемо довільну пару точок

$$
x=\left(x_{1}, \ldots, x_{n}\right), \quad y=\left(y_{1}, \ldots, y_{n}\right) \in \mathbb{H}^{n} .
$$

3 нерівності (2.2) отримаємо

$$
\langle x, y\rangle-f^{*}(y) \cap f(x)=\varnothing,
$$

тобто

$$
\langle x, y\rangle-f^{*}(y) \subset \mathbb{H}^{o} \backslash f(x),
$$

а тому

$$
\mathbb{H}^{o} \backslash\left(\langle x, y\rangle-f^{*}(y)\right) \supset f(x) .
$$

Взявши в останньому включенні перетин по всіх $y \in \mathbb{H}^{n}$, отримаємо такі включення

$$
\bigcap_{y \in \mathbb{H}^{n}}\left[\mathbb{H}^{o} \backslash\left(\langle x, y\rangle-f^{*}(y)\right)\right] \supset f(x),
$$

або

$$
\mathbb{H}^{o} \backslash \underset{y \in \mathbb{H}^{n}}{\cup}\left(\langle x, y\rangle-f^{*}(y)\right) \supset f(x) .
$$

Останнє включення означає, що $f \subset f^{* *}$. Теорему доведено.

Означення 2.5. Багатозначна функція $f: \mathbb{H}^{n} \rightarrow \mathbb{H}$ називається відкритою (відповідно, замкненою чи компактною), коли її графік є відкритою (відповідно, замкненою чи компактною) множиною в $\mathbb{H}^{n+1}$.

Теорема 2.6. Нехай $f: \mathbb{H}^{n} \rightarrow \mathbb{H}$ - багатозначна функція. Тоді спряжена до неї функція $f^{*}$ e лінійно опуклою. Якщо $f$-відкрита, то $f^{*}$ - замкнена.

Доведення. Значення спряженої функції можна записати у вигляді

$$
f^{*}(y)=\bigcap_{x \in \mathbb{H}^{n}}\left(\mathbb{H}^{o} \backslash(\langle x, y\rangle-f(x))\right) .
$$

При фіксованому $x$ функція

$$
y \longmapsto \mathbb{H}^{o} \backslash(\langle x, y\rangle-f(x))
$$

є багатозначною афінною функцією по $y$, а тому $\dddot{1}$ можна подати у вигляді

$$
y \longmapsto\langle x, y\rangle+\left[\mathbb{H}^{o} \backslash(-f(x))\right] .
$$

Отже $f^{*} \in$ перетином лінійно опуклих функцій виду (2.3), а тому за теоремою $1.8 f^{*}$ є лінійно опуклою функцією.

Крім того, якщо $f$ є відкритою, то кожна з функцій (2.3) є замкненою, а отже $f^{*}$ буде також замкненою. 
Означення 2.7. Лінійно опукла функція називається власною, якщо хоча $б$ для одного $x$ виконується співвідношення: $f(x) \cap \mathbb{H} \neq \varnothing$ і для всіх $x$ має місце нерівність $\mathbb{H} \backslash f(x) \neq \varnothing$.

Теорема 2.8. Нехай $f$-власна лінійно опукла функціл. Тоді $f^{*}-$ власна функиіл.

Доведення. Якщо $x_{0} \in E_{f}$, то

$$
f^{*}(y)=\mathbb{H}^{o} \backslash \underset{x \in \mathbb{H}^{n}}{\cup}(\langle x, y\rangle-f(x)) \subset \mathbb{H}^{o} \backslash\left(\left\langle x_{0}, y\right\rangle-f\left(x_{0}\right)\right) .
$$

Відповідно,

$$
\mathbb{H} \backslash f^{*}(y) \supset\left\langle x_{0}, y\right\rangle-f\left(x_{0}\right) \neq \varnothing
$$

для всіх $y$. 3 іншого боку, оскільки $f$ - власна лінійно опукла функція, то існує афінна функція $l(x)=\langle x, y\rangle+\alpha$, графік якої не перетинає $\Gamma(f)$. Тоді для цього $y$ матимемо, що

$$
(\langle x, y\rangle+\alpha) \cap f(x)=\varnothing,
$$

а отже

$$
(\langle x, y\rangle-f(x)) \cap-\alpha=\varnothing,
$$

і значить

$$
-\alpha \subset \mathbb{H}^{o} \backslash(\langle x, y\rangle-f(x)) .
$$

Toмy $f^{*}(y) \supset-\alpha \neq \varnothing$.

Наступна теорема [4] є гіперкомплексниим аналогом теореми Фенхеля-Моро.

Теорема 2.9. Нехай багатозначна функція $f: \mathbb{H}^{n} \rightarrow \mathbb{H}$ така, що

$$
\mathbb{H} \backslash f(x) \neq \varnothing
$$

для всіх $x \in \mathbb{H}^{n}$. Тодi $f^{* *}=f$ у тому $i$ лише у тому випадку, коли $f \in$ лінійно опуклою.

Доведення. Покажемо, що рівність $f^{* *}=f$ рівносильна лінійній опуклості функції $f$.

Якщо $f^{* *}=f$, то, згідно з теоремою 2.6, функція, спряжена до довільної функції, буде лінійно опуклою. Якщо $f\left(\mathbb{H}^{n}\right) \equiv \infty$, то рівність $f^{* *}=f$ отримується з формул (2.1) і (2.2). Маємо $f^{*}(y)=\mathbb{H}$ для всіх $y \in\left(\mathbb{H}^{n}\right)^{*}$ і $f^{* *}=\infty$. Оскільки $f \subset f^{* *}$ за теоремою 2.4, то достатньо показати, що для лінійно опуклої функції справедливе обернене включення $f \supseteq f^{* *}$. 
Нехай в деякій точці $x_{0}$ має місце нерівність $f\left(x_{0}\right) \neq f^{* *}\left(x_{0}\right)$. Тоді існуе афінна функція $l(x)=\left\langle x, y_{0}\right\rangle+\alpha$, така, що $\Gamma(l) \cap \Gamma(f)=\varnothing \mathrm{i}$ $w_{0}=\left\langle x_{0}, y_{0}\right\rangle+\alpha$, де $w_{0} \in f^{* *}\left(x_{0}\right) \backslash f\left(x_{0}\right)$. Тоді

$$
\begin{aligned}
f^{*}\left(y_{0}\right) & =\mathbb{H}^{o} \backslash \underset{x \in \mathbb{H}^{n}}{\cup}\left(\left\langle x, y_{0}\right\rangle-f(x)\right)= \\
& =\bigcap_{x \in \mathbb{H}^{n}}\left[\mathbb{H}^{o} \backslash\left(\left\langle x, y_{0}\right\rangle-f(x)\right)\right] \supsetneq(-\alpha),
\end{aligned}
$$

так як $\left[\left\langle x, y_{0}\right\rangle-f(x)\right] \neq-\alpha$ для всіх $x \in \mathbb{H}^{n}$. Для функції $f^{* *}$ справедливе включення

$$
\begin{aligned}
f^{* *}\left(x_{0}\right)= & \bigcap_{y \in\left(\mathbb{H}^{n}\right)^{*}}\left[\mathbb{H}^{o} \backslash\left(\left\langle x_{0}, y\right\rangle-f^{*}(y)\right)\right] \subset \\
& \subset \mathbb{H}^{o} \backslash\left(\left\langle x_{0}, y_{0}\right\rangle-f^{*}\left(y_{0}\right)\right) \subset \\
& \subset \mathbb{H}^{o} \backslash\left(\left\langle x_{0}, y_{0}\right\rangle+\alpha\right)=\mathbb{H}^{o} \backslash w_{0} .
\end{aligned}
$$

Тому $w_{0} \notin f^{* *}\left(x_{0}\right)$, що суперечить вибору точки $w_{0} \in f^{* *}\left(x_{0}\right) \backslash f\left(x_{0}\right)$.

Означення 2.10. Функція $f$ називається однорідною, якщо

$$
f(\lambda x)=\lambda f(x)
$$

для всіх скалярів $\lambda \in \mathbb{H} \backslash 0$.

Теорема 2.11. Нехай $f: \mathbb{H}^{n} \backslash \Theta \rightarrow \mathbb{H}, \Theta=(0,0, \ldots, 0) \in \mathbb{H}^{n}$, е власною лінійно опуклоюо однорідною фучнкиією $i f(\Theta)=\mathbb{H} \backslash 0$. Тоді $f$ є опорною функиією деякої множини.

Доведення. Розглянемо множину

$$
A=\left\{y \in \mathbb{H}^{n} \mid f(x) \not \supset\langle x, y\rangle \text { для всіх } x \in \mathbb{H}^{n}\right\}
$$

і покажемо, що

$$
f(x)=\mathbb{H}^{o} \backslash \bigcup_{y \in A}\langle x, y\rangle=W_{A}(x) .
$$

Якщо $y \in A$, то $\langle x, y\rangle \notin f(x)$ і $0 \notin\langle x, y\rangle-f(x)$ для всіх $x$. Відповідно,

$$
f^{*}(y)=\mathbb{H}^{o} \backslash \underset{x \in \mathbb{H}^{n}}{\cup}(\langle x, y\rangle-f(x))=\mathbb{H}^{o} \backslash\left(\mathbb{H}^{o} \backslash 0\right)=0 .
$$

Якщо $y \notin A$, то $\left\langle x_{0}, y\right\rangle \in f\left(x_{0}\right)$ для деякого $x_{0} \in \mathbb{H}^{n}, x_{0} \neq 0$. Тоді

$$
\begin{aligned}
f^{*}(y) & =\mathbb{H}^{o} \backslash \underset{x \in \mathbb{H}^{n}}{ }(\langle x, y\rangle-f(x))= \\
& =\mathbb{H}^{o} \backslash\left(f(\Theta) \underset{x \in \mathbb{H}^{n} \backslash \Theta}{\cup}(\langle x, y\rangle-f(x))\right)= \\
& =\mathbb{H}^{o} \backslash\left(\left(\mathbb{H}^{o} \backslash 0\right) \cup\left(\left\langle x_{0}, y\right\rangle-f\left(x_{0}\right)\right)\right)= \\
& =\mathbb{H}^{o} \backslash((\mathbb{H} \backslash 0) \cup 0)=\infty .
\end{aligned}
$$


Згідно з теоремою 2.8 одержуємо, що $f^{*} \in$ власною функцією. Тому $A \neq \varnothing \mathrm{i} f^{*}=\delta_{A}$. Беручи до уваги гіперкомплексну теорему ФенхеляМоро 2.9 і приклад 2.3, одержуємо рівність $f=f^{* *}=\delta_{A}^{*}=W_{A}$. Тобто $f$ - опорна функція множини $A$.

Наслідок 2.12. Якщо однорідна лінійно опукла функиія $f: \mathbb{H}^{n} \backslash \Theta \rightarrow \mathbb{H}$ є відмінною від афінної, то

$$
f^{*}(y)=\delta_{E_{f^{*}}}(y)
$$

Теорема 2.13. Якщ, $f: \mathbb{H}^{n} \backslash \Theta \rightarrow \mathbb{H}$ - однорідна лінійно опукла функиія, відмінна від афінної, то

$$
f(x)=\mathbb{H}^{o} \backslash \underset{y \in E_{f^{*}}}{\cup}\langle x, y\rangle .
$$

Доведення. Якщо $f$ - лінійно опукла функція, то за теоремою Фенхеля-Моро $f=f^{* *}=\delta^{*}$. Беручи до уваги приклад 2.3, переконуємось, що $f$ є опорною функцією множини $E_{f^{*}}$.

Означення 2.14. Нехай $f_{\alpha}: \mathbb{H}^{n} \rightarrow \mathbb{H}, \alpha \in A$, є багатозначними функціями. Функцію

$$
\left(\cup_{\alpha} f_{\alpha}\right)(x):=\bigcup_{\alpha} f_{\alpha}(x)
$$

назвемо об'єднанням функиій $f_{\alpha}$, а функцію

$$
\left(\bigcap_{\alpha} f_{\alpha}\right)(x):=\bigcap_{\alpha} f_{\alpha}(x)
$$

— ї перетином.

Для спряжених функцій має місце теорема двоїстості.

Теорема 2.15. Нехай $f_{\alpha}: \mathbb{H}^{n} \rightarrow \mathbb{H}, \alpha \in A$, е багатозначними бункиіями. Тоді виконуєтъся рівність

$$
\left(\cup_{\alpha} f_{\alpha}\right)^{*}=\bigcap_{\alpha} f_{\alpha}^{*}
$$

Доведення. 3 виразу (2.1) для спряжених функцій одержуємо

$$
\begin{aligned}
\left(\cup_{\alpha} f_{\alpha}\right)^{*}(y) & =\mathbb{H}^{o} \backslash \underset{x \in \mathbb{H}^{n}}{\cup}\left(\langle x, y\rangle-\cup_{\alpha} f_{\alpha}(x)\right)= \\
& =\mathbb{H}^{o} \backslash \bigcup_{x \in \mathbb{H}^{n}} \cup\left(\langle x, y\rangle-f_{\alpha}(x)\right)= \\
& =\mathbb{H}^{o} \backslash \bigcup_{\alpha} \bigcup_{x \in \mathbb{H}^{n}}\left(\langle x, y\rangle-f_{\alpha}(x)\right)= \\
& =\bigcap_{\alpha}\left(\mathbb{H}^{o} \backslash \cup_{x}\left(\langle x, y\rangle-f_{\alpha}(x)\right)\right)=\bigcap_{\alpha} f_{\alpha}^{*}(y) .
\end{aligned}
$$

Теорему доведено. 


\section{ЛiтерАтУРА}

[1] Ю. Б. Зелинский. Выпуклость. Избранные главы. К : Ін-т математики НАН України, 2012.

[2] И. Л. Кантор, А. С. Солодовников. Гиперкомплексные числа. М : Наука, 1973.

[3] Г. А. Мкртчян. О гиперкомплексно выпуклых множествах. К : ИМ АН УССР, 1987.

[4] М. В. Стефанчук, М. В. Ткачук. Лінійно опуклі та спряжені функції в гіперкомплексному просторі. Збірник праць Ін-ту математики НАН України, 12(3):225-235, 2015 .

Надійшла до редакцї̈ 12 серпня 2017, прийнята до друку 1 вересня 2017.

Стефанчук Марія Володимирівна

ІНСТИТУТ МАТЕМАТИКИ НАН УКРАїНИ, ВУЛ. ТЕРЕЩЕНКІвСЬКА, 3, м. КИїв, 01004, УКРAїHA

Email: mariast@imath.kiev.ua 